maternal and fetal acetaminophen concentrations were compared with those observed in the literature. umbilical cord data at birth.

Results The 3 different approaches to predicted acetaminophen PK in the umiblical vein were found to yield broadly similar results. Acetaminophen exposure was similar in maternal blood compared to venous umbilical cord blood. Prediction of the median dose fraction of acetaminophen converted to its metabolites $\left(f_{m}\right)$ revealed higher maternal acetaminophen-glucuronide formation clearance and sulphate formation compared to that in the fetal liver $\left(f_{m_{\text {_glucuronide }}} 52.2\right.$ vs $0 \%$ and $\mathrm{f}_{\mathrm{m} \_ \text {sulphate }} 30.4$ vs $0.8 \%$, respectively) and higher fraction of acetaminophen converted to the reactive metabolite $\mathrm{N}$-acetyl$p$-benzoquinone-imine $\left(f_{\mathrm{m} \_\mathrm{NAPQI}}, 6.5\right.$ vs $\left.0.06 \%\right)$ in pregnant women compared to their fetus.

Conclusion No differences were observed in the 3 approaches for integration of placental drug transfer. Differences in acetaminophen biotransformation to its metabolites between pregnant women and their fetuses were quantitatively predicted.

Disclosure(s) Paola Mian received a Short term Minor (STM2017) grant from the Stichting Sophia Kinderziekenhuis fonds to conduct this research.

\section{P67 IMPACT OF ENANTIOMER-SPECIFIC MATURATIONAL CHANGES IN PHARMACOKINETICS ON THE RACEMIC KETOROLAC TARGET TROUGH CONCENTRATION}

${ }^{1} \mathrm{M}$ Cloesmeijer, ${ }^{1}$ E Krekels, ${ }^{1} \mathrm{M}$ van Esdonk, ${ }^{2} \mathrm{~A}$ Lynn, ${ }^{3} \mathrm{~A}$ Smits, ${ }^{4} \mathrm{D}$ Tibboel, ${ }^{5} \mathrm{Y}$ Daali, ${ }^{6} \mathrm{~K}$ Olkkola, ${ }^{7,8} \mathrm{~K}$ Allegaert, ${ }^{4} \mathrm{P}$ Mian*. ${ }^{1}$ Systems Biomedicine and Pharmacology, Leiden Academic Centre for Drug Research, Leiden University, Leiden, The Netherlands; ${ }^{2}$ Department of Anesthesiology and Pain Medicine, Seattle Children's Hospital, University of Washington School of Medicine, Seattle, WA, USA; ${ }^{3}$ Neonatal Intensive Care Unit, University Hospitals Leuven, Leuven, Belgium; ${ }^{4}$ Department of Paediatric Surgery and Intensive Care, Erasmus MC - Sophia Childrens Hospital, Rotterdam, The Netherlands; ${ }^{5}$ Division of Clinical Pharmacology and Toxicology, Geneva University Hospitals, Geneva, Switzerland; ${ }^{6}$ Department of Anaesthesiology, Intensive Care and Pain Medicine, University of Helsinki and Helsinki University Hospital, Helsinki, Finland; ' Cluster Woman and Child, Department of Development and Regeneration, Biomedical Sciences, KU Leuven, Leuven, Belgium; ${ }^{8}$ Department of Pediatrics, Division of Neonatology, Erasmus MC - Sophia Childrens Hospital, Rotterdam, The Netherlands

\subsection{6/archdischild-2019-esdppp.105}

Introduction Ketorolac is a racemic drug with analgesic effects specific to its S-enantiomer. This study aimed to describe enantiomer-specific maturational pharmacokinetics (PK). Simulations were performed to describe how S-ketorolac exposure in infants differs from adults, and how this affects the adult racemic analgesic trough threshold $\mathrm{EC}_{50}$ (EC50 $0_{\text {thr-adult }}, 0.37$ $\mathrm{mg} / \mathrm{L})$ in infants $\left(\mathrm{EC} 50_{\text {thr-infant }}\right)$ when the same S-target is applied.

Methods A population PK analysis (NONMEM 7.3) was performed based on 1020 plasma samples from 5 studies including 80 patients (adults, children, infants) following single intravenous ketorolac administration.

Results S-ketorolac PK was best described with a 2-compartment model in infants and 3-compartment model in adults, while R-ketorolac PK was best described with a 2-compartment model in all. S-ketorolac clearance [mean population value: $3.45 \mathrm{~L} / \mathrm{h} / 56 \mathrm{~kg}$ ] and central volume of distribution (V1) [4.27 L/56kg] increased exponentially with bodyweight $(0.75$, 0.59 respectively). R-ketorolac clearance $[0.93 \mathrm{~L} / \mathrm{h} / 56 \mathrm{~kg}]$, peripheral volume of distribution (V2) and inter-compartmental clearance (Q) increased exponentially with bodyweight (0.62, 1.20, 0.76 respectively), V1 [4.11 L/56kg] linearly with bodyweight. Simulations revealed EC50 thr-adult $(0.37 \mathrm{mg} / \mathrm{L})$ contained $0.048 \mathrm{mg} / \mathrm{L} \mathrm{S}$-ketorolac as mean in typical adults

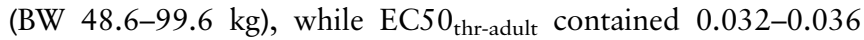
$\mathrm{mg} / \mathrm{L}$ S-ketorolac in typical infants (BW 5.3-10.6 kg). To reach adult $S$-enantiomer concentration $(0.048 \mathrm{mg} / \mathrm{L})$ in typical

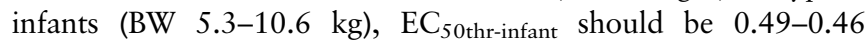
$\mathrm{mg} / \mathrm{L}$, respectively.

Conclusion Enantiomer-specific maturational PK of ketorolac were described. Subsequent simulations displayed differences in proportion of S- and R-ketorolac on the racemic threshold $\mathrm{EC}_{50}$. A The same S-ketorolac concentration necessitates a higher EC50 thr-infant to EC50 thr-adult

Disclosure(s) Nothing to disclose

\section{P68 SIGNIFICANT ORAL DRUG USE IN CRITICALLY ILL CHILDREN: RATIONAL THERAPY OR A BLACK BOX?}

${ }^{1,2}{ }^{\mathrm{M}}$ Mooij ${ }^{*},{ }^{3} \mathrm{~J}$ Windster, ${ }^{3} \mathrm{~J}$ van Rosmalen, ${ }^{4} \mathrm{~L}$ Hanff, ${ }^{3} \mathrm{D}$ Tibboel, ${ }^{3,5} \mathrm{~S}$ de Wildt. ${ }^{1}$ Erasmus MC - Sophia Childrens Hospital, Rotterdam; ${ }^{2}$ Willem-Alexander Children's Hospital, Leiden University Medical Centre, Leiden; ${ }^{3}$ Erasmus MC - Sophia Children's Hospital, Rotterdam; ${ }^{4}$ Princess Maxima Centre for Pediatric Oncology, Utrecht; ${ }^{5}$ Radboud University Medical Center, Nijmegen, The Netherlands

10.1136/archdischild-2019-esdppp.106

Background The disposition of orally prescribed drugs in critically ill children may be affected critical illness in in addition to age, resulting in erratic effects and safety. We aimed to study oral drug prescribing in the neonatal and pediatric intensive care unit (NICU and PICU).

Methods A one-year retrospective cohort study of all drug prescriptions, including route of administration for all children admitted to the NICU and PICU of the Erasmus MC-Sophia's children's hospital.

Results 1723 children with 2091 unique admissions received per admission (median [IQR]) 5 (3-10) drugs; 1 (0-2) orally and 3 (1-7) intravenously (IV). During mechanical ventilation $15 \%$ and $75 \%$ of drugs were given orally and IV, respectively. In non-ventilated patients, $27 \%$ of drugs were given orally and $60 \%$ IV. The 5 most frequently orally prescribed drugs were: vitamin $\mathrm{K}$, spironolactone, oral probiotics, amphotericin $\mathrm{B}$ (prophylaxis) and trimethoprim.

Discussion Critically ill infants receive a considerable proportion of drugs orally. Considering that critical illness may significantly impact intestinal drug absorption, this may expose them to an increased risk of ineffective or unsafe drug therapy.

Disclosure(s) Nothing to disclose

\section{P69 PHARMACOKINETICS OF A CONTINUOUS INFUSION OF PIPERACILLIN/TAZOBACTAM TO CHILDREN USING AN ELASTOMERIC PUMP (POPPET STUDY): PILOT DATA FROM DOUBLE LUMEN CENTRAL LINES}

\footnotetext{
${ }^{1,2} \mathrm{~J} \mathrm{Moss}^{*},{ }^{1} \mathrm{~V}$ Price, ${ }^{1,2} \mathrm{~S}$ Paulus, ${ }^{3} \mathrm{~V}$ Ramos-Martin, ${ }^{1} \mathrm{~A}$ Melaine, ${ }^{1} \mathrm{E}$ Evans, ${ }^{1} \mathrm{P}$ Newland, ${ }^{3} W$ Hope, ${ }^{1,2} \mathrm{D}$ Hawcutt, ${ }^{1,2} \mathrm{~B}$ Pizer. 'Alder Hey Children's NHS Foundation Trust; ${ }^{2}$ Department of Women's and Children's Health, Institute of Translational Medicine; ${ }^{3}$ Antimicrobial Pharmacodynamics and Clinical Pharmacology, University of Liverpool, Liverpool, UK
}

10.1136/archdischild-2019-esdppp.107

Background Piperacillin/Tazobactam is the first-line antibiotic for the treatment of febrile neutropenia in the UK. There is 